\title{
Errata: Feedback strategy on real-time multiple target tracking in cognitive vision system
}

\author{
Jie Shao \\ Tongji University \\ Department of Information and Communication \\ Engineering \\ Shanghai 201804, China \\ and \\ Shanghai University of Electric Power \\ Department of Computer and Information \\ Engineering \\ Shanghai 200090, China
}

\section{Zhen Jia}

United Technologies Research Center (China) Ltd. Shanghai 200120, China

\section{Zhipeng Li}

Fuqiang Liu

Tongji University

Department of Information and Communication

Engineering

Shanghai 201804, China

\section{Jianwei Zhao}

United Technologies Research Center (China) Ltd.

Shanghai 200120, China

Pei-Yuan Peng

LG Electronics China

R\&D Center

Shanghai, China

[DOI: 10.1117/1.3662423]

This article [Opt. Eng. 50, 107201 (2011)] was originally published on 28 September 2011 with an error in Pei-Yuan Peng's affiliation. The correct affiliation appears above.

The manuscript was corrected online on 2 November 2011. The article appears correctly in print. 